\title{
A novel technique to treat acquired Chiari I malformation after supratentorial shunting
}

\author{
Adriaan R. E. Potgieser ${ }^{1} \cdot$ Eelco W. Hoving ${ }^{1}$
}

Received: 29 May 2016/Accepted: 31 May 2016/Published online: 11 June 2016

(C) The Author(s) 2016. This article is published with open access at Springerlink.com

\begin{abstract}
Purpose The acquired Chiari I malformation with abnormal cranial vault thickening is a rare late complication of supratentorial shunting. It poses a difficult clinical problem, and there is debate about the optimal surgical strategy. Some authors advocate supratentorial skull enlarging procedures while others prefer a normal Chiari decompression consisting of a suboccipital craniectomy, with or without $\mathrm{C} 1$ laminectomy and dural patch grafting.

Methods We illustrate three cases of symptomatic acquired Chiari I malformation due to inward cranial vault thickening.

Results We describe a new surgical approach that appears to be effective in these patients. This approach includes the standard Chiari decompression combined with posterior fossa augmentation by thinning the occipital planum.

Conclusion Internal volume re-expansion of the posterior fossa by thinning the occipital planum appears to be an effective novel surgical strategy in conjunction with the standard surgical therapy of Chiari decompression.
\end{abstract}

Keywords Acquired Chiari I malformation .

Ventriculoperitoneal shunt $\cdot$ Cranial vault thickening

Adriaan R. E. Potgieser

a.r.e.potgieser@umcg.nl

1 Department of Neurosurgery, University Medical Center Groningen, Hanzeplein 1, P.O. Box 30.001, 9700

RB Groningen, The Netherlands

\section{Introduction}

The Chiari I malformation, or primary cerebellar ectopia, is one of the mildest forms of hindbrain herniation and is characterized by a downward displacement of the cerebellar tonsils through the foramen magnum into the cervical canal. The acquired Chiari I malformation is in fact a iatrogenic form of hindbrain herniation, first described after lumboperitoneal shunting $[2,4,8,17,21]$, but also after multiple lumbar punctures [18], after baclofen pump placement [19], and it was later recognized that an acquired Chiari I malformation can also occur after cystoperitoneal and ventriculoperitoneal shunting $[1,3,4,6,7,9-12,14,20]$.

In a previous case series, 17 of 1700 patients treated with ventriculoperitoneal shunting developed a Chiari I malformation as a late complication, of which only five patients were symptomatic [1]. It is therefore a rare complication of supratentorial shunting, but it poses a difficult problem. In many cases, the only symptom is headache, which is also a common problem in the general population. In these patients, it may point at overdrainage, slit ventricle syndrome, hydrocephalus, or acquired Chiari I malformation. There are some similarities between the described cases, but the causes of the Chiari I development seem to differ because there is a difference in timing of symptoms. Furthermore, there has been debate about the optimal surgical strategy to alleviate symptoms. We present three illustrative cases of an acquired Chiari I malformation due to cranial vault thickening as a perceived late complication of supratentorial shunting and introduce a novel surgical strategy.

\section{Case reports}

Case 1 is a 3-year-old boy with a bleeding in a left frontotemporal arachnoid cyst. He was treated with a craniotomy with 
fenestration and marsupialization of the arachnoid cyst. Half a year later, a subduroperitoneal drain without valve was implanted because of a persistent headache due to a large subdural hygroma at the location of the former arachnoid cyst (Fig. 1a). During the following years, the patient developed variable postural headaches attributed to liquor hypotension. Removing the drain led to immediate severe headache, and we decided to implant a new subduroperitoneal shunt with a PS medical medium valve. Subsequent shunt revisions over the years were performed with changing the valve to a Delta II, back to PS medical medium and finally to a Miethke 9/29. The latter valve resulted in an acceptable clinical condition (Fig. 1b) with symptoms of occasional mild headaches during the following years. At the age of 14 , he developed a different kind of headaches, characterized by short attacks of headaches relieved by vomiting. An MRI scan showed an acquired Chiari I malformation due to thickening of the cranial vault with a subsequent decrease of intracranial volume especially within the posterior fossa, without signs of syringomyelia or spinal CSF leakage (Fig. 1c). We decided to augment the volume of the posterior fossa in combination with decompression of the Chiari without creating a risk of a descending cerebellum. Therefore, we modeled the thickened occipital planum to a normal size and subsequently placed back the thinned bone. During the same procedure, a standardized C0 augmentation, $\mathrm{C} 1$ laminectomy, and a dural patch were given. The patient has remained without symptoms now for more than a year. A control MRI showed an adequate decompression (Fig. 1d). The volume of the posterior fossa was estimated on the T2-weighted scans using Brainlab iPlan 3.0 Cranial. The tentorium, occiput, McRae line, and clivus served as boundaries of the posterior fossa. There was an increase in the size of the posterior fossa $\left(188 \mathrm{~cm}^{3}\right.$ preoperatively, $205 \mathrm{~cm}^{3}$ postoperatively).

Case 2 is a 3-year-old girl with an increased intracranial pressure due to an aqueductal stenosis with hydrocephalus (Fig. 2a). We conducted a third ventriculostomy, which was complicated by a meningitis, external ventricular drainage, and eventually ventriculoperitoneal shunting (PS medical medium valve) with good result. Two years later, she developed persistent non-postural headaches. We decided to explore the ventriculostomy, which proved to be patent, and the ventriculoperitoneal shunt also functioned. After a period with mild headaches, she developed persistent headaches and a mild coordination disorder, which led to referral back to our clinic. A new MRI showed an acquired Chiari I malformation with clear cranial vault thickening (Fig. 2b) and a subsequent decrease in volume of the posterior fossa. A similar treatment strategy was chosen with augmentation of the posterior fossa by thinning the
Fig. 1 CT and MRI scans of a 3year-old boy with a bleeding in a left frontotemporal arachnoid cyst, treated with a craniotomy with fenestration and marsupialization of the cyst. A large left-sided subdural hygroma developed at the location of the former arachnoid cyst (a) after which a subduroperitoneal drain was placed with good result (b). At the age of 14 years, he developed an acquired Chiari I malformation due to cranial vault thickening (c), which was treated with a standardized $\mathrm{C} 0$ augmentation, $\mathrm{C} 1$ laminectomy, and a dural patch, followed by thinning of the occipital planum. The postoperative MRI shows an adequate decompression with enlargement of the posterior fossa (d)






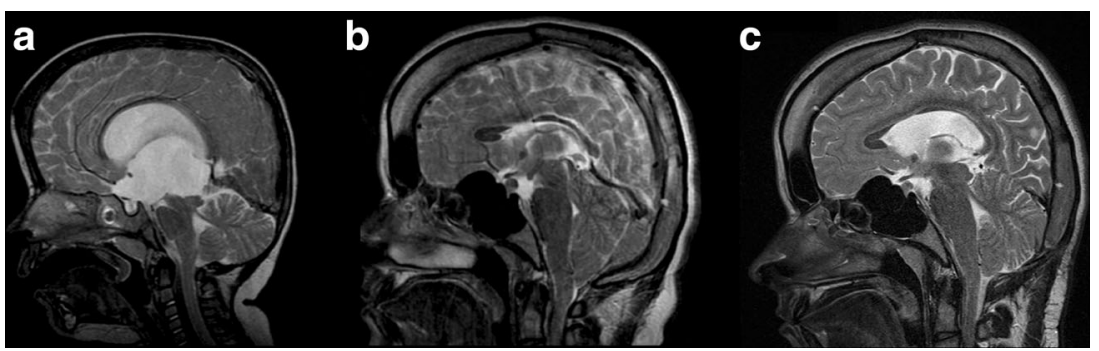

Fig. 2 MRI scans of a 3-year-old girl with hydrocephalus due to aqueductal stenosis (a). She was treated with a third ventriculostomy and a ventriculoperitoneal shunt. Several years later, she developed an acquired Chiari I malformation with cranial vault thickening (b). After augmentation of the posterior fossa by thinning the occipital planum and decompression of $\mathrm{C} 0$ and a $\mathrm{C} 1$ laminectomy with dural patch grafting, the postoperative MRI scan shows an adequate decompression (c)

occipital planum and decompression of $\mathrm{C} 0$ and a $\mathrm{C} 1$ laminectomy with dural patch grafting. We also decided to upgrade the valve to a gravitational Miethke 10/30. The posterior fossa volume increased (from $155 \mathrm{~cm}^{3}$ preoperatively to $174 \mathrm{~cm}^{3}$ postoperatively). The patient's headaches have diminished ever since. MRI showed an adequate decompression with a subtle increase in the size of the lateral ventricles (Fig. 2c).

Case 3 is a boy with X-linked recessive chondrodysplasia punctata (mutation in the CDPX1 gene), which is a very rare disease characterized by skeletal dysplasias [5]. Although not very well known, types of chondrodysplasia punctata can affect the craniocervical junction [14]. At the age of 1 month, he presented with a raised intracranial pressure due to a bleeding in a cyst in the right thalamus (Fig. 3a). We performed a third ventriculostomy with fenestration of the cyst and left an

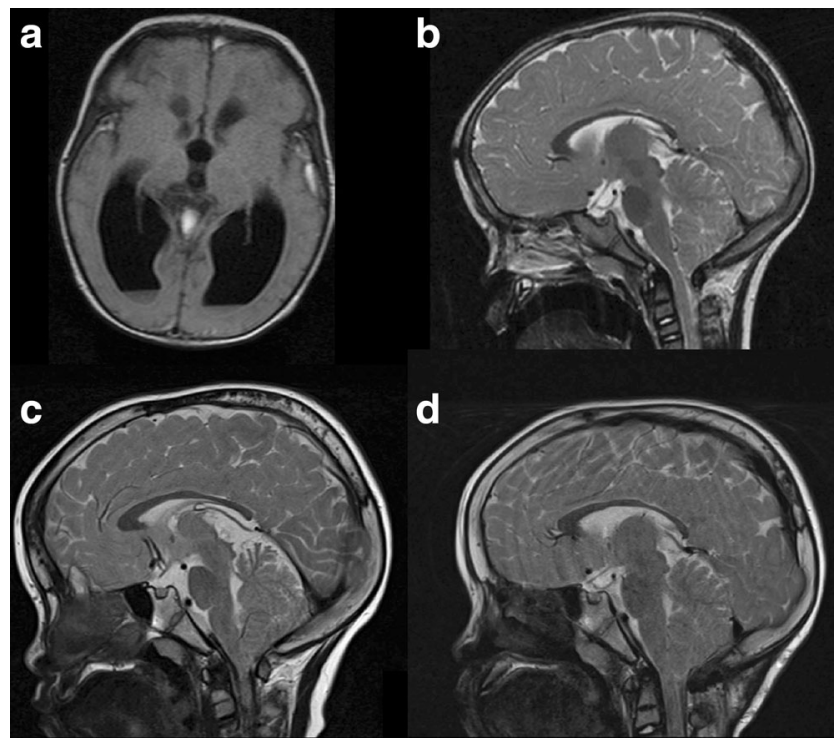

Fig. 3 Case 3. MRI scan of a bleeding from a cyst in the right thalamus (a), treated with fenestration of the cyst, external ventricular drainage, and eventually a ventriculoperitoneal shunt. During follow-up, an asymptomatic Chiari I malformation was seen (b), that became symptomatic after a clear thickening of the cranial vault in the following years (c). The postoperative MRI scan shows an adequate decompression after a $\mathrm{C} 1$ laminectomy and posterior fossa decompression, during which we placed back the thinned occipital plane after remodeling it to a normal size (d) external ventricular drain. Two weeks after removal of the drain, the patient developed a raised intracranial pressure, and a ventriculoperitoneal shunt with PS medical low valve was inserted. During the following years, he had intermittent periods of non-postural headaches. A Chiari I malformation was diagnosed, but this was considered an asymptomatic finding (Fig. 3b). At the age of 6 years, the valve was upgraded and replaced by a Miethke 9/24. Half a year later, the boy had short paroxysmal non-postural headaches, and the MRI scan showed thickening of the cranial vault with an "acquired" Chiari I malformation (Fig. 3c). We performed a similar procedure as the previous two cases. A $\mathrm{C} 1$ laminectomy and posterior fossa decompression were performed in combination with thinning of the occipital planum (Fig. 3d). After this surgical procedure, the patient was immediately relieved from his symptoms. The posterior fossa volume increased (from $114 \mathrm{~cm}^{3}$ preoperatively to $127 \mathrm{~cm}^{3}$ postoperatively).

\section{Discussion}

There has been a continuing debate about the optimal surgical treatment of the acquired Chiari I malformation. Some authors prefer supratentorial skull enlarging procedures $[1,4,11,12]$, which can be considered a major surgical procedure with potentially more morbidity than our approach. Others advocate a suboccipital craniectomy, with or without $\mathrm{C} 1$ laminectomy and dural patch grafting $[9,13]$, which can be considered as a standard procedure for the "normal" Chiari I malformation. In our cases, the Chiari I malformation coincides with acquired thickening of the skull with a subsequent decrease in volume of the posterior fossa. Our novel surgical approach to the acquired Chiari I malformation with inward skull thickening and subsequent loss of posterior fossa volume includes a standard Chiari decompression $(\mathrm{C} 0 / \mathrm{C} 1$ decompression and dural patch grafting) combined with internal posterior fossa volume augmentation by thinning the occipital planum. In order to improve conditions for proper 
shunting, we tried to augment the size of the ventricles by inserting gravitational shunts in combination with the local treatment of the Chiari and posterior fossa as described. The combined surgical procedure of Chiari decompression and internal volume expansion of the posterior fossa by thinning of the occipital planum appears to be effective in our three cases, without creating new problems of a symptomatic descending cerebellum.

Based on the literature, three mechanisms of an acquired Chiari I malformation can be differentiated due to a difference in timing of symptoms. For both supratentorial shunting and lumbar shunting, early-onset cases (roughly within a year of placement) without cranial vault thickening have been described [6, 10, 17-20], while it is also seen as a late complication as was also the case in our patients $[1,3,4,12,13]$.

An early-onset acquired Chiari I after lumboperitoneal shunting appeals to the pressure gradient theory [21]. A craniospinal pressure gradient is generated which basically sucks the cerebellum into the cervical canal. The successful treatment of an acquired Chiari I malformation caused by lumboperitoneal shunting by converting it to a ventriculoperitoneal shunt supports this theory [4, 17]. An early-onset acquired Chiari I after supratentorial shunting must have a different mechanism because no downward pressure gradient is generated. This may be caused by overdrainage of the ventricles, which leads to sagging of the brain as a whole, possibly in combination with a small (but previously sufficiently sized) posterior fossa. Our three cases had a late-onset development of the Chiari I malformation, characterized by modifications of the skull induced by the shunt. Initially named cephalocranial disproportion [7], Osuagwu et al. called it posterior cranial fossa disproportion, because they showed that there are normal cerebellar and supratentorial volumes in shunted children but a smaller posterior cranial fossa [16]. They propose that the etiology is an arrested posterior cranial fossa growth, similar to the etiology in the "normal" adult Chiari I malformation [15, 16]. However, they do not describe an abnormally thickened and inward growing cranial vault, as in our patients and those of many others [1]. It is possibly the combination of an arrested skull growth with subsequent inward cranial vault thickening due to chronic overdrainage that gives rise to this kind of acquired Chiari I malformation [1]. This seems evident in our cases, and our surgical approach appeals to this problem.

\section{Conclusion}

We illustrate three cases of symptomatic acquired Chiari I malformation with cranial vault thickening as a perceived late complication of supratentorial shunting. Internal volume reexpansion of the posterior fossa by thinning the occipital planum appears to be an effective novel surgical strategy in conjunction with the standard surgical therapy of Chiari decompression.

\section{Compliance with ethical standards}

Disclosures No financial disclosures.

Conflict of interest The authors report no conflict of interest concerning the findings specified in this paper.

Open Access This article is distributed under the terms of the Creative Commons Attribution 4.0 International License (http:// creativecommons.org/licenses/by/4.0/), which permits unrestricted use, distribution, and reproduction in any medium, provided you give appropriate credit to the original author(s) and the source, provide a link to the Creative Commons license, and indicate if changes were made.

\section{References}

1. Caldarelli M, Novegno F, Di Rocco C (2009) A late complication of CSF shunting: acquired Chiari I malformation. Childs Nerv Syst 25:443-452

2. Chumas PD, Armstrong DC, Drake JM, Kulkarni AV, Hoffman HJ, Humphreys RP et al (1993) Tonsillar herniation: the rule rather than the exception after lumboperitoneal shunting in the pediatric population. J Neurosurg 78:568-573

3. Di Rocco C, Tamburrini G (2003) Shunt dependency in shunted arachnoid cyst: a reason to avoid shunting. Pediatr Neurosurg 38:164

4. Di Rocco C, Velardi F (2003) Acquired Chiari type I malformation managed by supratentorial cranial enlargement. Childs Nerv Syst 19:800-807

5. Gupta N, Ghosh M, Shukla R, Das GP, Kabra M (2012) Brachytelephalangic chondrodysplasia punctata: a case series to further delineate the phenotype. Clin Dysmorphol 21:113-117

6. Hassounah MI, Rahm BE (1994) Hindbrain herniation: an unusual occurrence after shunting an intracranial arachnoid cyst. Case report. J Neurosurg 81:126-129

7. Hoffman HJ, Tucker WS (1976) Cephalocranial disproportion. A complication of the treatment of hydrocephalus in children. Childs Brain 2:167-176

8. Johnston I, Jacobson E, Besser M (1998) The acquired Chiari malformation and syringomyelia following spinal CSF drainage: a study of incidence and management. Acta Neurochir (Wien) 140: 417-427, discussion 427-8

9. Kim SK, Cho BK, Chung YN, Kim HS, Wang KC (2002) Shunt dependency in shunted arachnoid cyst: a reason to avoid shunting. Pediatr Neurosurg 37:178-185

10. Lazareff JA, Kelly J, Saito M (1998) Herniation of cerebellar tonsils following supratentorial shunt placement. Childs Nerv Syst 14: 394-397

11. Martinez-Lage JF, Ruiz-Espejo AM, Almagro MJ, Alfaro R, Felipe-Murcia M, Lopez-Guerrero AL (2009) CSF overdrainage in shunted intracranial arachnoid cysts: a series and review. Childs Nerv Syst 25:1061-1069

12. Martinez-Lage JF, Ruiz-Espejo Vilar A, Perez-Espejo MA, Almagro MJ, de San Pedro JR, Felipe Murcia M (2006) Shuntrelated craniocerebral disproportion: treatment with cranial vault expanding procedures. Neurosurg Rev 29:229-235 
13. Mendonca R, Lima TT, Oppitz PP, Raupp SF (2006) Herniation of the cerebellar tonsils after suprasellar arachnoid cyst shunt: case report. Arq Neuropsiquiatr 64:523525

14. Menezes AH, Vogel TW (2008) Specific entities affecting the craniocervical region: syndromes affecting the craniocervical junction. Childs Nerv Syst 24:1155-1163

15. Nishikawa $M$, Sakamoto H, Hakuba A, Nakanishi N, Inoue Y (1997) Pathogenesis of Chiari malformation: a morphometric study of the posterior cranial fossa. J Neurosurg 86: $40-47$

16. Osuagwu FC, Lazareff JA, Rahman S, Bash S (2006) Chiari I anatomy after ventriculoperitoneal shunting: posterior fossa volumetric evaluation with MRI. Childs Nerv Syst 22:14511456
17. Payner TD, Prenger E, Berger TS, Crone KR (1994) Acquired Chiari malformations: incidence, diagnosis, and management. Neurosurgery 34:429-434, discussion 434

18. Sathi S, Stieg PE (1993) “Acquired" Chiari I malformation after multiple lumbar punctures: case report. Neurosurgery 32:306-309, discussion 309

19. Tubbs RS, Law C, Oakes WJ, Grabb PA (2004) Acquired Chiari I malformation following baclofen pump placement in a child. Case report. J Neurosurg 101:211-213

20. Udayakumaran S (2009) Post CSF shunting Chiari I malformation-sequel or complication? Hypertension or hypotension? Childs Nerv Syst 25:1391-1392, author reply 1393

21. Welch K, Shillito J, Strand R, Fischer EG, Winston KR (1981) Chiari I "malformations" - an acquired disorder? J Neurosurg 55: 604-609 